\title{
Regulation of macrophage immunometabolism in atherosclerosis
}

\author{
Graeme J. Koelwyn', Emma M. Corr', Ebru Erbay $\circledast^{2,3,4}$ and Kathryn J. Moore ${ }^{1 \star}$
}

\begin{abstract}
After activation, cells of the myeloid lineage undergo robust metabolic transitions, as well as discrete epigenetic changes, that can dictate both ongoing and future inflammatory responses. In atherosclerosis, in which macrophages play central roles in the initiation, growth, and ultimately rupture of arterial plaques, altered metabolism is a key feature that dictates macrophage function and subsequent disease progression. This Review explores how factors central to the plaque microenvironment (for example, altered cholesterol metabolism, oxidative stress, hypoxia, apoptotic and necrotic cells, and hyperglycemia) shape the metabolic rewiring of macrophages in atherosclerosis as well as how these metabolic shifts in turn alter macrophage immuneeffector and tissue-reparative functions. Finally, this overview offers insight into the challenges and opportunities of harnessing metabolism to modulate aberrant macrophage responses in disease.
\end{abstract}

M acrophages are essential components of mammalian tissues and are strategically positioned throughout the body to maintain tissue homeostasis and act as immune sentinels. Macrophages can be either embryonically seeded in tissues, where they are maintained through self-renewal, or derived from monocyte precursors, which infiltrate tissues and differentiate in response to their microenvironment. Regardless of their origin, macrophages are remarkable in their plasticity: they can change their functional phenotype in response to local environmental cues and consequently execute a diverse set of functions essential to host defense and tissue repair. Such functions include phagocytosis of apoptotic cells and pathogens, elaboration of immune-effector molecules and growth factors, and remodeling of the extracellular matrix. To fulfill these effector functions, macrophages must coordinate a plethora of intrinsic cellular processes to meet environmental demand. It is becoming increasingly appreciated that the rewiring of metabolic pathways and the repurposing of metabolic intermediates to facilitate appropriate extrinsic cellular responses are central to this intrinsic coordination.

Early studies of metabolic reprogramming in cancer cells provided a window into the understanding of how metabolism can regulate cell fate. Elevated uptake of glucose and secretion of lactate are metabolic hallmarks of highly proliferative tumor cells, which rely heavily on glycolysis even when sufficient oxygen is present to support mitochondrial oxidative phosphorylation (OXPHOS) - so called 'aerobic glycolysis' or the 'Warburg effect'1 . Whereas aerobic glycolysis is much less efficient than OXPHOS at generating ATP, this metabolic pathway is nonetheless well suited for proliferation because it allows metabolic intermediates to be siphoned off for biosynthesis of nucleotides, lipids, and proteins needed in the dividing cell. Similar metabolic alterations have been identified in some immune cells, including macrophages, as they transition from rested to activated states ${ }^{1}$ to meet cellular demands, such as phagocytosis, proliferation, and cytokine production ${ }^{2,3}$. This metabolic modulation can result in energy generation; production of building blocks for cell maintenance and proliferation; and modulation of cellular signaling ${ }^{4}$. Studies of macrophages polarized in vitro to either a classically activated (by using lipopolysaccharide (LPS) and the cytokine IFN $\gamma$ ) or an alternatively activated (by using the cytokine IL-4) state have shown that metabolic reprogramming of macrophages not only is critical for energy homeostasis but also directly influences cell functions. Although these in vitro phenotypes have been useful models to study, relatively little is known about how macrophage metabolism changes in vivo, when cells encounter a multitude of signals in the tissue microenvironment during either pathogen challenge or sterile inflammatory responses.

During atherosclerosis, sterile inflammation is set in motion by the intramural retention of cholesterol-rich lipoproteins in large- and medium-sized arteries (reviewed in ref. ${ }^{5}$ ). Imbalances in cellular and systemic cholesterol homeostasis promote the artery-wall deposition of lipoproteins containing the apolipoprotein apoB, particularly in areas of altered blood flow that disturb the normally quiescent endothelium. These sequestered lipoproteins are susceptible to various modifications (for example, oxidation, enzymatic cleavage, and aggregation) that render them proinflammatory; the modified lipoproteins then trigger the recruitment of monocyte-derived cells into the subendothelial space. There, monocytes differentiate into macrophages that clear the accumulated lipoproteins, a process that, although initially beneficial, ultimately results in the accumulation of cholesterolladen macrophage foam cells that promote plaque formation. These macrophage foam cells have a diminished migratory capacity $^{6,7}$ and elicit production of proinflammatory cytokines and chemokines that amplify the immune response by recruiting reinforcements, including additional monocytes, $\mathrm{T}$ cells, and neutrophils (reviewed in ref. ${ }^{8}$ ). For reasons that remain poorly understood, the resulting low-grade inflammation is nonresolving, and plaque progression can continue over decades of life ${ }^{9}$. As plaques advance, macrophages proliferate ${ }^{10}$, secrete inflammatory mediators (for example, cytokines and proteases), and ultimately die from lipid and metabolic stresses ${ }^{11}$, thereby releasing their lipid contents and other inflammatory debris that contributes 
to the formation of a necrotic core. In plaques, macrophages are exposed to a variety of proinflammatory cytokines, oxidized lipids, cholesterol crystals, oxidative stress, and danger-associated molecular patterns derived from dying cells; these biomolecules together create a complex microenvironment that perpetuates macrophage inflammation ${ }^{5}$.

Even after optimal medical treatment for atherosclerotic risk factors with current antihypertensive and cholesterol-lowering drugs (for example, statins), substantial residual cardiovascular disease risk remains ${ }^{12}$. There is thus high interest in targeting the inflammatory component of atherosclerosis and developing strategies to alter the inflammatory phenotype of plaque macrophages ${ }^{8,13}$. The encouraging finding, from a recent large clinical trial, that antibody-mediated inhibition of the proinflammatory cytokine IL- $1 \beta$ can decrease cardiovascular events in high-risk populations ${ }^{14}$ has provided optimism that such a strategy may work. This possibility has fueled interest in understanding the immunometabolic pathways that influence macrophage inflammatory responses during atherosclerosis and whether these pathways in turn might represent new therapeutic targets for intervention. Recent studies have begun to elucidate how macrophage metabolism of glucose, cholesterol, fatty acids, and amino acids is rewired in atherosclerosis, and how these changes shape both ongoing and future inflammatory responses. It is already clear that the complex plaque microenvironment results in a variety of activation modes in macrophages and consequently in a more complex and heterogeneous rewiring of metabolic pathways than seen in classically or alternatively activated macrophage phenotypes.

This Review provides a framework for understanding the metabolic programs that influence macrophage activation and inflammatory phenotypes; how these programs are affected by microenvironmental factors in the atherosclerotic plaque; the role of metabolism in the epigenetic programming of future inflammatory responses; and whether these various pathways can be targeted for therapeutic intervention in atherosclerosis.

\section{An overview of cellular metabolic programs}

Metabolic pathways play a major role in immune-cell function. The intertwined pathways of glycolysis, the tricarboxylic acid (TCA) cycle, the pentose-phosphate pathway (PPP), fatty acid oxidation (FAO), fatty acid synthesis, and amino acid metabolism serve to generate energy, produce building blocks necessary for cellular maintenance and proliferation, and modulate cellular signaling (in-depth review in ref. ${ }^{15}$ ). Below, we provide a brief description of these pathways, how they are interconnected, and how they intersect in regulating macrophage activation and effector function.

In macrophages, the glucose transporter GLUT-1 initiates uptake of glucose, which is then phosphorylated by hexokinase to glucose 6-phosphate, which in turn is metabolized in glycolysis or in the PPP. During glycolysis, processing of glucose in the cytosol yields two net ATPs as well as pyruvate, which either is converted by lactate dehydrogenase to lactate or enters the TCA cycle. Glycolysis yields two molecules of ATP per molecule of glucose and also provides biosynthetic intermediates that fuel the PPP and fatty acid synthesis. The final glycolytic enzyme, pyruvate kinase (PKM1 or PKM2), regulates glycolytic flux by alternating between catalytically active tetrameric or slow dimeric states, thereby regulating funneling of earlier glycolytic intermediates into the PPP or into serinebiosynthesis pathways ${ }^{16}$

The PPP is an anabolic pathway that runs parallel to glycolysis and generates ribose 5-phosphate for synthesis of nucleotides and $\mathrm{NADPH}$, an essential cofactor in lipid biosynthesis and the production of antioxidants, nitric oxide, and reactive oxygen species (ROS). Macrophages require high levels of PPP flux and NADPH during respiratory bursts both to eliminate extracellular bacteria and to synthesize antioxidants such as glutathione and thioredoxin, which limit oxidative damage to cells. In contrast, PPP flux is limited in alternatively activated macrophages ${ }^{17}$, which perform tissue-reparative functions and produce less ROS. PPP-derived NADPH is also used for de novo fatty acid synthesis to expand the endoplasmic reticulum (ER) and Golgi to support enhanced cytokine secretion.

OXPHOS diverges from glycolysis after the production of pyruvate, which is shuttled to the mitochondria and enters the TCA cycle. The TCA cycle converts pyruvate or fatty acids into acetyl coenzyme A (acetyl-CoA), which is condensed with oxaloacetate, thereby forming citrate. This important intermediate is then either further oxidized by the TCA cycle or hydrolyzed by ATP-citrate lyase to generate oxaloacetate and cytosolic acetyl-CoA, the latter of which contributes to protein acetylation and the synthesis of cholesterol and fatty acids needed for new membranes. The TCA cycle produces $\mathrm{NADH}$ and flavin adenine dinucleotide $\left(\mathrm{FADH}_{2}\right)$, which transfer electrons to the electron-transport chain to support OXPHOS yielding ATP. The TCA cycle and OXPHOS are often associated with quiescent or nonproliferative cells.

FAO is the most fruitful producer of cellular ATP, wherein a single fatty acid molecule such as palmitate can yield over $100 \mathrm{~mol}-$ ecules of $\mathrm{ATP}^{15}$. Short-chain fatty acids can diffuse passively into the mitochondria for oxidation, whereas medium- and long-chain fatty acids are imported into the mitochondria through conjugation to carnitine palmitoyltransferase 1 . FAO yields a number of products including acetyl-CoA, $\mathrm{NADH}$, and $\mathrm{FADH}_{2}$, which can used by the TCA cycle and electron-transport chain to generate ATP. In contrast, fatty acid synthesis utilizes products derived from other metabolic pathways, including glycolysis, the TCA cycle, and PPP, to provide lipids necessary for differentiation and proliferation ${ }^{18,19}$. Fatty acid synthesis involves the activity of enzymes including sterol regulatory element-binding protein, fatty acid synthase, and acetyl-CoA carboxylase to convert metabolic intermediates, such as TCA-cycle-derived citrate or glycolysis-derived glycerol, into triacylglycerols and phospholipids, which are key components of cellular structures.

Metabolism of amino acids can have important roles in cell effector functions. For example, glutamine feeds the TCA cycle, in which it can be used either for ATP production or as a source of citrate for fatty acid synthesis, whereas arginine and tryptophan can be metabolized to support cellular proliferation and anabolic growth. Indeed, metabolism of arginine by either inducible nitric oxide synthase or arginase- 1 was initially used to define classically and alternatively activated macrophage subsets, respectively ${ }^{20}$.

\section{The metabolic phenotypes of acutely activated macrophages}

The aforementioned metabolic pathways are intricately dependent on one another during macrophage activation to synergistically fulfill cell effector functions. For many years, in vitro and in vivo activation of macrophages, and the study of their metabolic and effector responses, focused on two activation phenotypes: 'M1' macrophages classically activated by LPS and IFN $\gamma$ stimulation, and alternatively activated 'M2' macrophages activated by IL- 4 stimulation (hereafter referred to as M[LPS+IFN $\gamma]$ and M[IL-4], respectively). Although it has become clear that these macrophage phenotypes represent extremes, their mechanistic exploration provides a starting point for understanding how macrophage activation and metabolism intersect in dictating cellular responses (Fig. 1).

LPS induces a profound metabolic rewiring of macrophages and dendritic cells, which is characterized by increased glucose uptake and enhanced aerobic glycolysis, and is coincident with impaired OXPHOS via the TCA cycle ${ }^{21,22}$, a response resembling the Warburg effect in highly proliferative tumor cells ${ }^{1}$. In those cells, pyruvate produced by the glycolytic pathway is metabolized to lactate and 


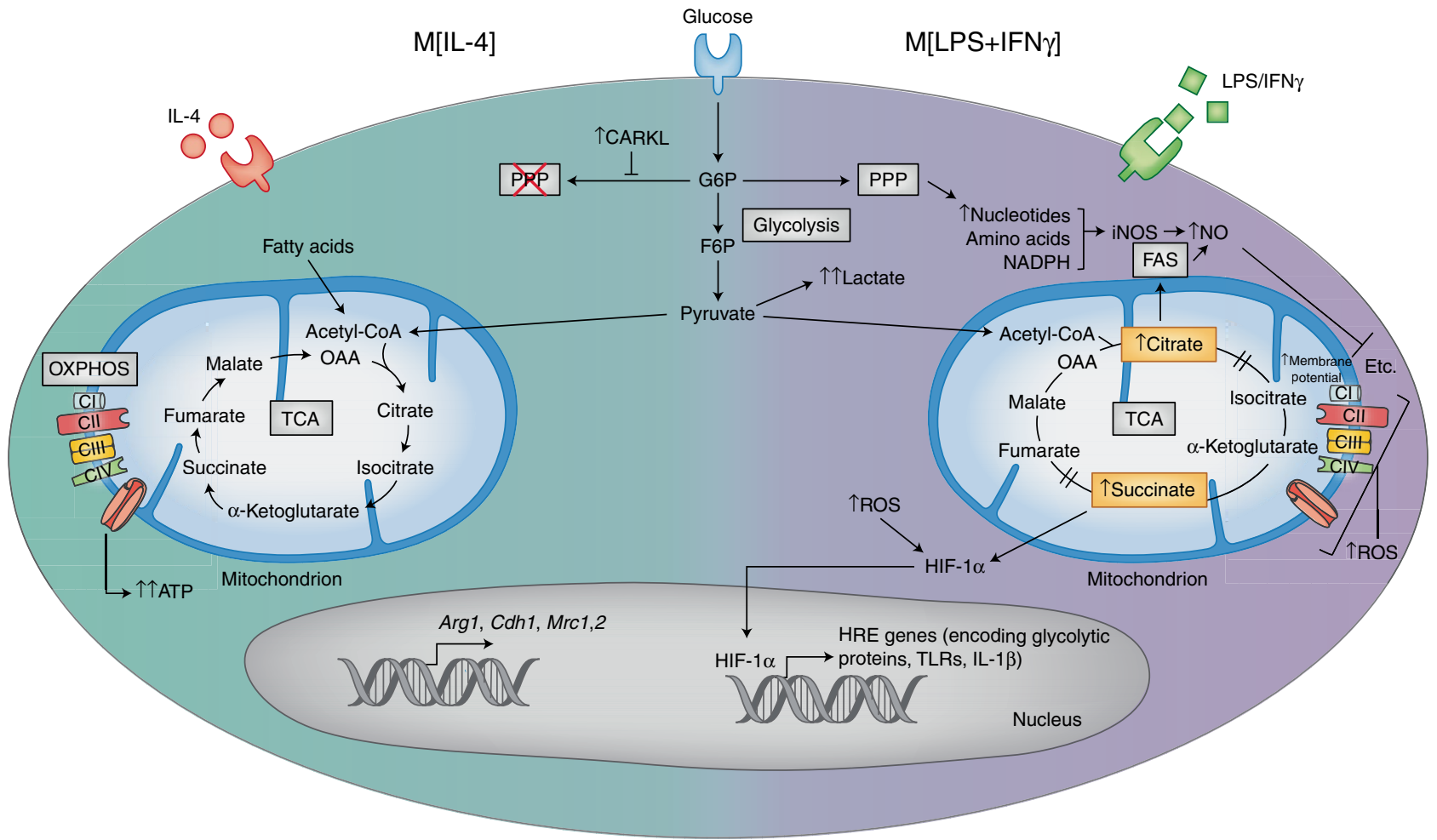

Fig. 1 | Metabolic pathways controlling macrophage activation states. Glycolysis involves the conversion of glucose molecules into various metabolic byproducts, culminating in the end product pyruvate as well as two net ATPs. In macrophages treated with IL-4 (M[IL-4], left side of the cell) pyruvate (and fatty acids) enters the intact TCA cycle as acetyl-CoA, thus resulting in sustained ATP production via OXPHOS and leading to the upregulation of genes associated with tissue repair. In contrast, in macrophages treated with LPS and IFN $\gamma$ (M[LPS+IFN $\gamma$, right side of the cell), most pyruvate is converted into lactate and secreted. Furthermore, the enzyme CARKL is downregulated, and as a result, glycolysis also feeds the PPP, thereby generating nucleotides, amino acids, and NADPH. The TCA cycle is broken in two places in M[LPS+IFN $\gamma$ ] macrophages, thus resulting in the accumulation of citrate, which in turn is used to drive fatty acid synthesis (FAS) and succinate, which stabilizes the transcription factor HIF-1 $\alpha$. HIF-1 $\alpha$ enters the nucleus and promotes the expression of hypoxia response element (HRE)-containing genes, which encode both glycolytic and proinflammatory proteins such as IL-1 $\beta$ ). The disturbed TCA cycle and decreased OXPHOS also result in increased levels of ROS, which further stabilize HIF-1 $\alpha$, allowing it to drive glycolysis and inflammatorygene expression. G6P, glucose 6-phosphate; F6P, fructose 6-phosphate; OAA, oxaloacetate. Credit: Kim Caesar/Springer Nature

secreted, while TCA cycle intermediates accumulate, thus resulting in decreased OXPHOS and direct effects on immune responses ${ }^{22,23}$. For example, succinate accumulation leads to stabilization of the transcription factor HIF- $1 \alpha$ and upregulation of enzymes involved in glycolysis and of the proinflammatory cytokine IL-1 $\beta$ (ref. ${ }^{22}$ ). Citrate is shuttled to the cytosol, where it is used to generate fatty acids for membrane biogenesis and prostaglandin production ${ }^{24}$, and to produce the antimicrobial metabolite itaconic acid ${ }^{25}$. Other TCAcycle intermediates, such as fumarate, contribute to macrophage 'innate immune memory' by modifying epigenetic remodeling by KDM5 demethylases ${ }^{26}$ and consequently influences future immune responses, as discussed later in this Review.

The PPP is enhanced in M[LPS+IFN $\gamma]$ macrophages through increased flux of glucose intermediates through the pathway and downregulation of the kinase CARKL ${ }^{17}$, thus allowing for increased NADPH synthesis. NADPH availability from the PPP is essential for cholesterol metabolism and fatty acid synthesis ${ }^{27,28}$, which supports phagocytosis ${ }^{18,19}$, as well as for the expansion of the Golgi and ER that is required for production of inflammatory cytokines $^{29,30}$. M[LPS+IFN $\left.\gamma\right]$ macrophages also exhibit elevated lipid accumulation via enhanced fatty acid uptake via the scavenger receptor CD36 and diminished triglyceride lipolysis ${ }^{28}$. In addition, amino acid metabolism modulates immune-cell functions in M[LPS+IFN $\gamma]$ macrophages: glutamine is required for LPS-induced IL- $1 \beta$ secretion $^{31}$, whereas arginine is metabolized by inducible nitric oxide synthase, thus producing nitric oxide, which acts both as an antimicrobial agent and as a signal for vasodilation, angiogenesis, and insulin secretion. The extent to which other metabolites can alter macrophage functional states is only beginning to be appreciated.

Compared with M[LPS+IFN $\gamma]$ macrophages, M[IL-4] macrophages have a less well-understood metabolic phenotype. M[IL-4] macrophages exhibit a precipitous increase in FAO and OXPHOS that is thought to contribute to anti-inflammatory responses ${ }^{23}$. CD36-mediated uptake of triglycerides and subsequent lipolysis via lysosomal lipase feeds the TCA cycle and increases OXPHOS, thereby sustaining M[IL-4] polarization ${ }^{32}$. However, the specific role of FAO in M[IL-4] macrophages has recently been questioned in studies showing that etomoxir-mediated inhibition of FAO or deficiency in CPT2, an enzyme required for fatty acid import, does not alter the M[IL-4] phenotype ${ }^{33,34}$. Notably, M[IL-4] macrophages do depend on glucose to drive OXPHOS ${ }^{23}$, because inhibition of glycolysis suppresses IL-4-induced gene expression ${ }^{35}$; however, the downregulation of the PPP limits glucose flux into this pathway ${ }^{17}$. In terms of amino acid metabolism, glutamine flux into both the TCA cycle and hexosamine pathway promotes M[IL-4] macrophage polarization $^{23}$. Furthermore, arginine metabolism by arginase-1 produces L-ornithine, which is broken down into polyamines and L-proline to support macrophage proliferation and for collagen production used in tissue repair ${ }^{36,37}$.

Recent studies have revealed that the metabolic rewiring of $\mathrm{M}[\mathrm{LPS}+\mathrm{IFN} \gamma]$ and M[IL-4] macrophages does not represent 
a metabolic blueprint for all activated macrophages. Different microbial stimuli, endogenous danger ligands, and tissue microenvironmental signals lead to specific and complex metabolic rewiring of macrophages. As these unique metabolic signatures of macrophages have been unraveled, it has become clear that the type and fate of nutrients used are linked not only to the macrophage's need for energy but also to the generation of metabolites that act as signaling molecules and building blocks (for example, lipids and nucleic acids) needed for cellular maintenance or proliferation.

Studies using agonists of Toll-like receptors (TLRs) other than TLR4 or using whole microbial lysates suggest that the Warburg metabolism of M[LPS+IFN $\gamma$ ] macrophages is not a universal response to pathogenic stimuli ${ }^{2}$. In vitro stimulation of human CD14 ${ }^{+}$monocytes with ligands of TLR2 (with its ligands Pam3Cys or P3C) or TLR3 (with its ligand poly(I:C)), or with whole pathogen lysates from Escherichia coli, Staphylococcus aureus, or Mycobacterium tuberculosis, induces increases in glycolysis and $\mathrm{OXPHOS}^{2}$. These metabolic responses are connected to specific functional activities; blocking glycolysis with 2-deoxyglucose restricts production of IL1 $\beta$ in response to LPS and P3C, whereas blocking OXPHOS with rotenone lowers IL1 $\beta$ production and phagocytosis only in P3C-stimulated cells.

Cytokine stimulation of monocytes and macrophages also induces a distinct metabolic rewiring that is only beginning to be understood. As described for IL-4, stimulation with the cytokine IL-13, which also polarizes macrophages toward alternative activation, increases mitochondrial biogenesis, FAO and OXPHOS ${ }^{38,39}$. Stimulation of macrophages with the myeloid growth factors M-CSF or GM-CSF increases $\left[{ }^{3} \mathrm{H}\right] 2$-deoxyglucose uptake and oxygen consumption rate. Interestingly in mouse peritoneal macrophages, M-CSF is a stronger inducer of the glycolytic extracellular acidification rate and lactate production than GM-CSF, whereas the opposite is true in human monocyte-derived macrophages ${ }^{40}$ and mouse bone marrow-derived macrophages ${ }^{41}$. The functional consequences of M-CSF- and GM-CSF-induced macrophage metabolism on cytokine production, phagocytosis, and cell migration remain to be determined. Interestingly, in human monocytes, IFN $\gamma$ alone can enhance glycolysis and lactate production ${ }^{42}$, yet stimulation of mouse peritoneal macrophages with IFN $\gamma$ in combination with other cytokines (for example, TNF $^{39}$ or TNF plus IL-1 $\beta$ (ref. ${ }^{43}$ )) does not alter $\left[{ }^{3} \mathrm{H}\right] 2$-deoxyglucose uptake. Additionally, IL-10 promotes anti-inflammatory effects by suppressing the kinase $\mathrm{mTOR}^{44}$, regulating GLUT-1 translocation to inhibit glycolytic flux, and promoting mitophagy of dysfunctional mitochondria to help preserve respiratory capacity.

The divergent macrophage effector responses to in vitro TLR, pathogen, and cytokine stimuli described above highlight that macrophage cellular responses to environmental cues probably require different functional outputs accomplished through specific metabolic rewiring. Although these studies of macrophage metabolic responses in vitro have led to an appreciation of the versatility and extent to which metabolic rewiring regulates the inflammatory function of these cells, macrophages in vivo are exposed to multiple stimuli simultaneously and must mount a coordinated response to balance a multitude of cellular demands, each dependent on specific energy and metabolic-intermediate requirements. In the next section, we discuss how macrophage metabolism is dynamically regulated within the multifaceted microenvironment of atherosclerotic plaques. Specifically, we consider how priming of macrophage precursors by the systemic atherosclerotic milieu may alter cell function before recruitment to plaques, and how plaque microenvironmental stimuli, such as lipoproteins, cholesterol, hypoxia and oxidative stress, apoptotic and necrotic cell death, and hyperglycemia, may regulate macrophage metabolism within this dynamic microenvironment (Fig. 2).

\section{Monocyte priming in hematopoietic reservoirs and the circulation in atherosclerosis}

Myeloid cells experience complex signals in vivo as they transit from hematopoietic reservoirs into different tissues, where they undergo differentiation into macrophages in unique microenvironments. Monocytes, which are derived from reservoir populations in the bone marrow and spleen, then released into the circulation, are the major precursors of the atherosclerotic-plaque macrophage population (reviewed in ref. ${ }^{5}$ ). Before their arrival in the artery wall, these monocytes are exposed to a variety of pathologic stimuli within the systemic milieu. Evidence from studies of monocytes isolated from people with atherosclerosis and from mouse models of atherosclerosis indicates that this exposure modulates both cellular metabolism and function, thus priming monocytes toward an inflammatory phenotype well before their differentiation within the plaque microenvironment.

Levels of monocytes in the circulation, particularly those of the $\mathrm{CD}_{14}{ }^{+}$subpopulation in humans and the Ly6C $\mathrm{C}^{\text {hi }}$ subpopulation in mice, are strongly correlated with atherosclerosis progression ${ }^{8}$. Multiple factors in the atherosclerotic systemic milieu have been shown to drive an increase in myelopoiesis, including cholesterol homeostasis ${ }^{45}$, chronic inflammation, activation of the sympathetic nervous system ${ }^{46,47}$, and defective cholesterol efflux ${ }^{48}$. Enhanced glucose uptake by monocytes and hematopoietic precursor cells has been observed in mouse models of atherosclerosis and is thought to contribute to enhanced myelopoiesis. Inhibition of GLUT-1 directly, or by increasing high-density lipoprotein (HDL) levels, decreases myleoproliferation in mouse models ${ }^{49}$. Using a transplant approach in which atherosclerosis-susceptible $A p o e^{-/-}$mice were reconstituted with either wild-type or $A p o e^{-/-}$bone marrow, Sarrazy et al. ${ }^{50}$ observed enhanced uptake of $\left[{ }^{14} \mathrm{C}\right] 2$-deoxyglucose in the bone marrow, spleen, and plaques of $A$ poe $^{-1-}$ bone marrow transplanted mice. Within the bone marrow compartment, the mice with $A$ poe $e^{-/-}$bone marrow exhibited elevated glucose uptake by multipotent progenitors, abundance of granulocytic-macrophage progenitors (GMPs), and increased GLUT-1 expression and uptake of the fluorescent D-glucose analog 2-NBDG by hematopoietic stem and progenitor cells (HSPCs). These alterations in glucose utilization were accompanied by enhanced oxygen consumption and increased mitochondrial membrane potential. Mechanistically, the upregulation of GLUT-1 was found to be dependent on signaling by the interleukin receptor IL-3R $\beta$, which drove subsequent glycolytic substrate utilization by the mitochondria and accelerated myelopoiesis in $\mathrm{Apoe}^{-/-}$mice.

These metabolic alterations also extend to monocytes isolated from atherosclerotic patients. In a study comparing human monocytes isolated from healthy individuals or individuals with atherosclerosis, Shirai et al. ${ }^{51}$ have mechanistically explored the concept of 'monocyte priming' in relation to cellular metabolic status. After ex vivo stimulation with LPS and IFN $\gamma$, monocytes isolated from the atherosclerotic patients produced more IL- 6 and IL- $1 \beta$ than did monocytes from control individuals, and this primed phenotype was maintained after their in vitro differentiation into macrophages. Together with these differences, LPS+IFN $\gamma$-stimulated monocytes from atherosclerotic patients exhibited a higher oxygen consumption rate and respiratory reserve capacity, as well as higher glycolytic extracellular acidification rate and glycolytic flux, than did monocytes from healthy individuals. Notably, the enhanced glucose uptake of atherosclerotic monocytes was proportional to mitochondrial ROS production, oxidative stress, and subsequent inflammatory signaling. Molecular-level investigation of the underlying mechanisms identified a glucose-ROS-PKM2-STAT3 pathway through which glucose utilization led to unbalanced ROS generation from the mitochondrial respiratory chain, which in turn induced the redox-sensitive enzyme PKM2 (one of the isoforms of the enzyme that catalyzes the final step of glycolysis and converts 


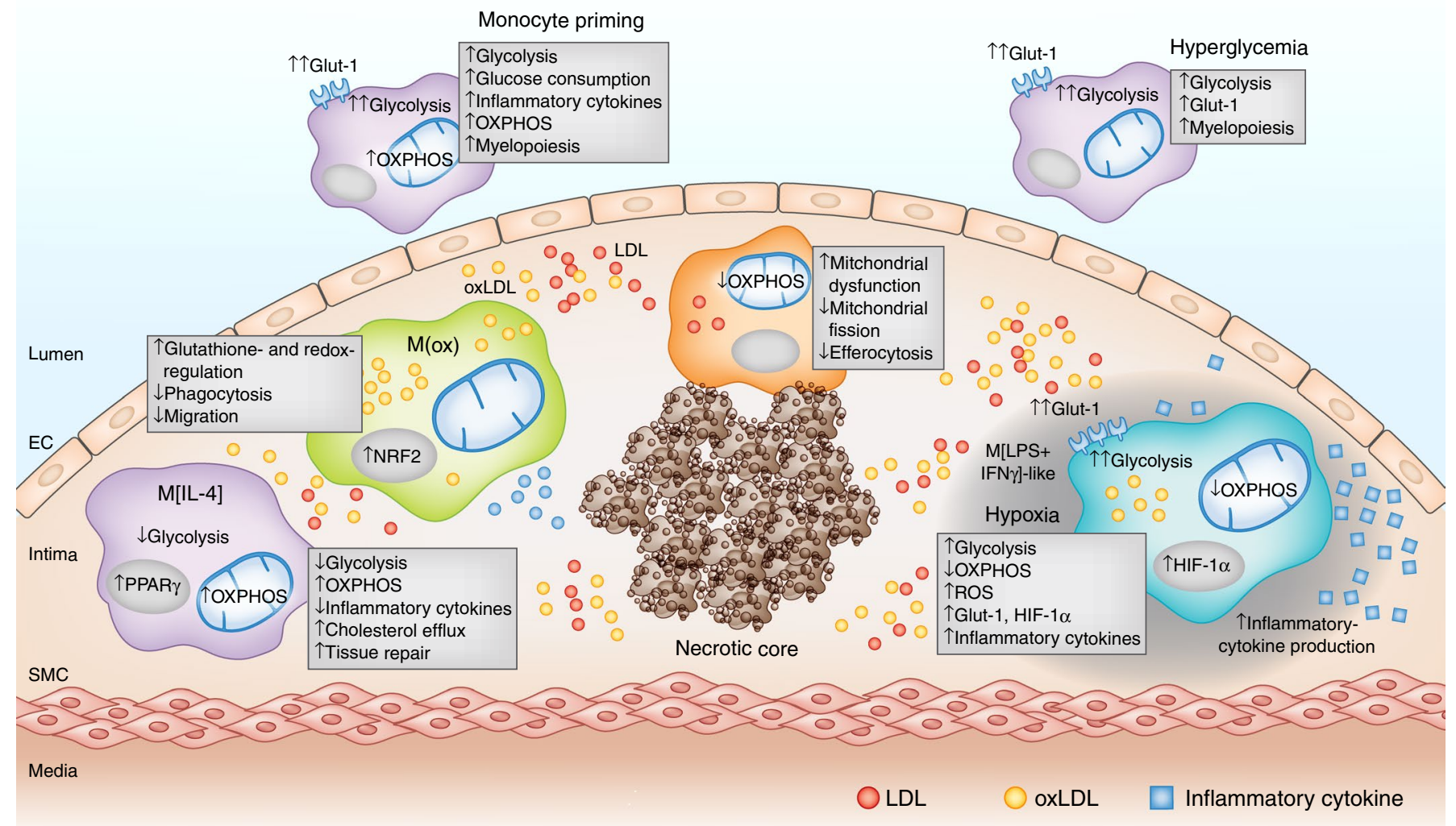

Fig. 2 | Macrophage metabolic reprogramming in atherosclerotic plaques. In the hypercholesterolemic systemic environment, monocytes exhibit a 'primed' inflammatory phenotype characterized by enhanced inflammatory responses that correspond to increases in both glycolysis and OXPHOS. After entering into the plaque microenvironment, a variety of stimuli including lipids, cytokines, cell apoptosis and necrosis, hypoxia, and hyperglycemia can influence macrophage metabolic reprogramming and subsequent function. Distinct macrophage phenotypes have been identified within plaques, including M[IL-4]-like, M(ox), M[LPS+IFN $\gamma$ ]-like, and hypoxia-associated macrophages. These different types of macrophages are associated with distinct metabolic signatures and inflammatory functions. PPAR $\gamma$, peroxisome proliferator-activated receptor gamma; NRF2, nuclear factor 2. Credit: Kim Caesar/ Springer Nature

phosphoenolpyruvate to pyruvate) to translocate from the cytoplasm to the nucleus, thus resulting in activation of the transcription factor STAT3 and transcription of IL6 and IL1B.

Cumulatively, the preclinical and clinical evidence to date provides direct evidence that the atherosclerotic systemic milieu primes both reservoir and circulating hematopoietic myeloid populations before recruitment to the plaque microenvironment. Specifically, these cells utilize more glucose, have enhanced rates of glycolysis and OXPHOS, and are hyperinflammatory before recruitment to plaques.

\section{Microenvironmental cues shape macrophage metabolism and function in plaques}

In atherosclerotic vessels, as in other tissue sites, distinct microenvironmental factors influence macrophage metabolism and phenotype, and these forces change as plaques evolve and progress. As described below, the environment of atherosclerotic plaques is influenced by hyperlipidemia, oxidative stress, hypoxia, and cell death. In addition, in people with diabetes, hyperglycemia, a comorbid condition that accelerates atherosclerosis, is also a major factor influencing macrophage metabolism. Together, these microenvironmental signals are important drivers of the metabolic reprogramming of macrophages and of their inflammatory responses, both of which ultimately affect disease progression and plaque stability. Below, we summarize in vivo evidence, and support from in vitro mechanistic work, of plaque microenvironmental features that drive changes in macrophage function alongside metabolic adaptations and consequently facilitate cellular effector responses in atherosclerosis.

Macrophage uptake of modified lipoproteins and resulting phenotypes. Monocyte-derived and tissue-resident macrophages lead the response to lipoproteins that are retained and modified in the artery wall. These macrophages attempt to clear the inflammatory lipids and are transformed into 'foamy' macrophages as they store the excess cholesterol and triglyceride in cytoplasmic lipid droplets. Cholesterol loading of macrophages diminishes their migratory capacity, and, for reasons that are still unclear, these macrophage foam cells persist in the artery wall, where they foster chronic inflammation. In vitro studies mimicking macrophage foam cell formation by using oxidized low-density lipoprotein (oxLDL) have shown that oxLDL is a potent inducer of macrophage glycolysis $^{52}$, inflammation ${ }^{53}$, and mitochondrial oxidative damage ${ }^{54}$. Both oxLDL and its associated oxidized phospholipids have been shown to induce inflammatory signaling via TLR4 homodimers or TLR4-TLR6 heterodimers, thus leading to cytokine and chemokine expression as well as changes in the actin cytoskeleton ${ }^{55,56}$. In addition, the unregulated uptake of oxLDL via CD36 results in the nucleation of intracellular cholesterol crystals that destabilize lysosomes, thereby impairing cholesterol and fatty acid metabolism, and activating the NLRP3 inflammasome and production of IL-1 $\beta$ (refs ${ }^{57,58}$ ). Numerous cellular metabolic disturbances, all present in 
plaque macrophages, have been linked to NLRP3-inflammasome activation, including the release of lysosomal cathepsins, mitochondrial ROS, and extracellular ATP ${ }^{59}$. Furthermore, free-cholesterol enrichment of macrophage foam cell membranes can enhance inflammatory signaling from lipid rafts, particularly via activation of TLR4 signaling and the transcription factor NF- $\kappa B$, independently of ligand stimulation ${ }^{60-62}$. In addition, oxLDL can activate HIF-1 $\alpha$, which fuels increased glucose uptake, glycolysis, and macrophage inflammatory responses ${ }^{52,63}$. Thus, macrophage foam cells generated in vitro exhibit multiple characteristics of M[LPS+IFN $\gamma$ ] macrophages, including elevated glycolysis, TLR4 activation, and IL- $1 \beta$ production.

Attempts to characterize the plaque macrophage inflammatory phenotype in vivo have revealed multiple subsets of macrophages, which are dynamically regulated during the progression and regression of disease. Macrophages with general markers of the M[LPS+ IFN $\gamma$ ] phenotype are present in early atherosclerotic lesions, and their proportion increases as plaques progress to more complex inflammatory lesions ${ }^{64}$. These M[LPS+IFN $\gamma$ ]-like macrophages are enriched in lipid, in agreement with an increase in glycolysis, and they populate different regions within plaques than macrophages with markers of the M[IL-4] phenotype ${ }^{65}$. During atherosclerosis regression, the balance of M[LPS+IFN $\gamma]$-like and M[IL-4]like macrophages switches, such that M[IL-4]-like macrophages become more prominent ${ }^{66-68}$. Plaque macrophages resembling the M[IL-4] phenotype show little lipid accumulation, in agreement with a high level of FAO, and these cells are thought to be atheroprotective because of their anti-inflammatory and profibrotic properties. Indeed, treatment of $L d l r^{-1-}$ mice with the M[IL-4]-polarizing cytokine IL-13 inhibits atherosclerosis progression ${ }^{69}$. In addition, a third macrophage phenotype, termed $\mathrm{M}$ (ox), has been identified in atherosclerotic plaques and found to be induced in vitro in response to oxidized phospholipids ${ }^{70} . \mathrm{M}(\mathrm{ox})$ macrophages are characterized by high expression of genes whose transcription is dependent on the transcription factor NRF2 (including Hmox1, Srxn1, and Txnrd1), and by a glutathione- and redox-regulating phenotype. These macrophages have lower phagocytic and migratory capacity than do macrophages stimulated with M[IL-4] or M[LPS+IFN $\gamma$, characteristics that may potentially contribute to their persistence in plaques. Attempts to quantify these macrophage populations in advanced plaques of $L d l r^{--}$mice have indicated a distribution of $\sim 40 \%$ classically activated $\left(\mathrm{CD} 86^{\mathrm{hi}}\right), \sim 20 \%$ alternatively activated $\left(\mathrm{CD} 206^{+}\right)$, and $\sim 30 \% \mathrm{M}(\mathrm{ox})$ macrophages.

To date, metabolic phenotyping of plaque macrophages beyond cell-surface markers has been limited, not only because of the challenges of isolating macrophages from plaques (typically through aortic digestion, which can alter the cellular metabolic state) but also because of previous technological limitations in understanding cellular phenotypes at the single-cell level. Within plaques, it is likely that discrete phenotypic populations and/or a phenotypic spectrum of atherosclerosis-induced macrophage activation states exist, which are driven by the various plaque microenvironmental signals encountered in a spatiotemporal fashion. Indeed, emerging and established single-cell and imaging technologies hold promise in identifying, characterizing, and understanding these spatiotemporal activation states within plaques, thus enabling subsequent targeting of either distinct phenotypic populations or shifting the phenotypic spectrum for therapeutic benefit.

Macrophage cholesterol storage and efflux. Under homeostatic conditions, lipoproteins taken up by macrophages are transported to late endolysosomal compartments, where cholesterol esters are hydrolyzed. The endolysosomal proteins NPC1 and NPC2 function together to transport free cholesterol to the cytoplasm, where it is trafficked to the cell membrane for export or delivered to the ER for reesterification and storage in lipid droplets. Macrophages in advanced plaques show evidence of massive free-cholesterol accumulation, thus suggesting a breakdown in these processes that maintain cholesterol homeostasis. Excess free-cholesterol accumulation is damaging to membranes and causes dysregulation of metabolic processes that occur in the ER and mitochondria.

Cholesterol efflux via the lipid transporters ABCA1 and ABCG1 is key to maintaining cholesterol homeostasis in plaque macrophages and promoting reverse cholesterol transport to the liver for excretion $^{71}$. This process relies on proper mitochondrial function. A sustained supply of ATP is required to fuel the activity of ABC transporters to move their substrates (free cholesterol and phospholipids) across the membrane to an acceptor, and treatment of macrophages with oligomycin, which blocks the production of ATP from OXPHOS, markedly decreases cholesterol efflux to apoA1 (ref. ${ }^{72}$ ). Furthermore, macrophages from mice lacking PGC1 $\alpha$, a master regulator of mitochondrial biogenesis, have impaired cholesterol efflux ${ }^{72}$, and accordingly $L d l r^{-1-}$ mice deficient in hematopoietic Ppargcla expression exhibit accelerated progression of atherosclerosis $^{73}$. Mitochondria are also an important source of oxysterol ligands for the liver $\mathrm{X}$ receptor transcription factors, which coordinate the expression of genes whose products are involved in the response to cholesterol excess, including ABCA1 and ABCG1. The mitochondrial sterol 27-hydroxylase CYP27A1 converts excess cholesterol to 27-hydroxycholesterol, and mitochondrial transport of cholesterol from the outer to inner membrane is rate limiting in CYP27A1's generation of oxysterols.

Studies of hypercholesterolemic mouse models suggest that mitochondrial metabolism is disturbed in atherosclerotic-plaque macrophages. In the REVERSA mouse model of genetically reversible hypercholesterolemia, mitochondrial genes have been found to be significantly downregulated in the aorta during progression of atherosclerosis, coincident with rapid lesion expansion and elevated $\mathrm{CD}^{+} 8^{+}$macrophage content within plaques ${ }^{74}$. Functional enrichment analysis indicated an overrepresentation of mitochondrial and ROS homeostasis pathways, and markedly diminished expression of genes involved in mitochondrial biogenesis and the antioxidant response (for example, Sod2, Ucp1, and Ucp3). Notably, after genetic reversal of hypercholesterolemia, more than 200 genes were found to be upregulated in the aorta, $30 \%$ of which were nuclear-encoded mitochondrial genes. Of those mitochondrial cholesterol-responsive genes, $70 \%$ corresponded to genes downregulated during hypercholesterolemia and lesion expansion, thus suggesting that this effect can be reversed. Although this study did not examine isolated macrophages, given their prominence in mouse atherosclerotic plaques, macrophage mitochondrial function may be speculated to drive this response.

Strategies that increase mitochondrial biogenesis or function have been shown to enhance the export of cholesterol from macrophages. In atherosclerotic-plaque macrophages, microRNA (miR)33 appears to be a nexus for repressing mitochondrial function, cholesterol efflux, and FAO. miR-33a and miR-33b are upregulated in human and mouse atherosclerotic plaques ${ }^{68,72}$, and these noncoding RNAs post-transcriptionally inhibit genes in networks regulating mitochondrial biogenesis and function (Ppargc1a, Slc25a3, $P d k 4$, and $N r f 1)^{72}$, intracellular cholesterol transport and efflux (NPC1 (in humans only), OSBPL6, ABCA1, and Abcg1 (in mice only) $)^{75,76}$, autophagy and lipid-droplet catabolism (ATG5, ATG12, $L C 3 B$, and $L A M P 1)^{77,78}$, and FAO (HADHB, CPT1A, CROT, and PRKAA1 $)^{79}$. Antagonism of miR-33 in mouse models of atherosclerosis has been shown to be atheroprotective through raising plasma levels of HDL cholesterol, promoting macrophage cholesterol efflux and reverse cholesterol transport, enhancing mitochondrial function, and decreasing macrophage inflammatory polarization ${ }^{68,72,80,81}$.

Oxidative stress and hypoxia. Oxidative stress and inflammation are interrelated processes that form a robust feed-forward 
cycle that fuels atherosclerotic-plaque progression. During atherosclerosis, macrophage ROS are generated through the actions of mitochondrial oxidative metabolism, NADPH oxidases, peroxidases, nitric oxide synthases, cyclooxygenases, and lipoxygenases. The macrophage antioxidant response is key to decreasing cellular levels of ROS and protecting mitochondria and other organelles, proteins, and nucleic acids from oxidative damage; however, the transcription of antioxidant genes and mitochondrial transport of the antioxidant glutathione are suppressed in plaque macrophages, thus magnifying inflammation in the artery wall. For example, NADPH oxidase-derived ROS from macrophages promotes oxidation of LDL in the artery wall, amplifying macrophage foam cell formation $^{82}$. Furthermore, excessive mitochondrial ROS generated as a by-product of electron-transport-chain reactions can damage mitochondrial DNA, proteins, and lipids, and these processes increase during atherosclerosis in mice ${ }^{83}$. The oxidation or release of mitochondrial DNA can also serve as a danger-associated molecular pattern that activates multiple innate immunological signaling pathways in macrophages, including the CpG DNA receptor TLR9, the NLRP3 inflammasome ${ }^{84,85}$, and the cyclic GMP-AMP synthase-stimulator of interferon genes pathway ${ }^{86-88}$. Decreasing mitochondrial oxidative stress in macrophages can prevent inflammation in plaques and decrease atherosclerotic burden in mice ${ }^{54}$.

In addition to cellular oxidative stress, the imbalanced demand and supply of oxygen in the plaque microenvironment leads to hypoxia in human and mouse atherosclerotic plaques and is a key contributor to atherogenesis ${ }^{89}$. Local hypoxia within the plaque environment arises because of a combination of increased cellular metabolic demand coupled with impaired oxygen delivery due to increased diffusion distance within plaques ${ }^{90}$. In humans, hypoxic regions in the plaque microenvironment are rich in HIF- $1 \alpha$ -expressing macrophages and foam cells ${ }^{91}$, which are associated with angiogenesis and plaque hemorrhage ${ }^{90}$. In atherosclerotic mice, a decrease in plaque hypoxia after administration of hyperoxic carbogen gas $\left(95 \% \mathrm{O}_{2}, 5 \% \mathrm{CO}_{2}\right)$ results in favorable changes in plaque characteristics, including an increase in M[IL-4]-like macrophages and a decrease in both IL- 6 production and necrotic-core size ${ }^{92}$.

ROS and hypoxia are potent drivers of macrophage metabolic reprogramming that results in enhanced reliance on glycolysis. Critical to this metabolic switch is activation of the HIF- $1 \alpha$ transcription factor, which induces the expression of GLUT-1 and glycolytic enzymes (for example, HK, PFK, and PFKB3), and limits $\mathrm{OXPHOS}^{93}$. Hypoxia response element-containing genes also encode a variety of inflammatory proteins, such TLR2, TLR4, IL-1 $\beta$, and CXCR4, thus resulting in a coordinated inflammatory response. As such, activation of the HIF- $1 \alpha$ pathway, particularly in macrophages, is critically involved in modulating both the metabolic and inflammatory effector responses. Increased glucose uptake associated with HIF-pathway activation has been demonstrated in mouse and human atherosclerotic lesions. In human plaques, expression of HIF- $1 \alpha$ colocalizes with high expression of GLUT1 (official symbol SLC2A1), GLUT3 (official symbol SLC2A3), HK1, and $H K 2$ in macrophage-rich regions ${ }^{94}$, thus suggesting enhanced glycolysis in these cells. Furthermore, expression of the HIF- $1 \alpha$ -regulated cytokine IL- $1 \beta$ is also elevated within macrophage-rich hypoxic plaque regions ${ }^{94}$, and hypoxia potently induces NLRP3 activation and IL-1 $\beta$ expression in human macrophages. Similarly, in the plaques of $L d l r^{-/-}$and $A p o e^{-/-}$mice, there is an abundance of hypoxic HIF-1 $\alpha$-expressing macrophages ${ }^{90,95}$, and laser capture of HIF- $1 \alpha$-positive macrophages has revealed significantly greater Glut1 (Slc2a1) expression than that in macrophages from normoxic regions. In vitro studies of macrophage foam cells support these in vivo observations. Hypoxia markedly potentiates macrophage glycolytic flux in the presence of proatherogenic mediators such as oxLDL and proinflammatory cytokines (IL-1 $\beta$, IFN $\gamma$, and TNF) ${ }^{63}$, and this effect is dependent on HIF- $1 \alpha$ and PFKFB3. Interestingly, the glycolytic shift in response to hypoxia relies on mitochondria for appropriate oxygen sensing, because macrophages that lack mitochondria do not accumulate HIF- $1 \alpha$ in response to hypoxia ${ }^{96}$. HIF- $1 \alpha$ also increases macrophage sterol and triglyceride content by stimulating sterol synthesis and suppressing cholesterol efflux via ABCA1 (ref. ${ }^{95}$ ), and consequently further exacerbates cholesterol accumulation in plaque macrophages. Macrophages deficient in HIF-1 $\alpha$ exhibit low expression of inflammatory genes (for example, those encoding monocyte chemoattractant protein-1 and osteopontin), as well as apoptosis, and hematopoietic deficiency of HIF- $1 \alpha$ (ref. ${ }^{90}$ ) or GLUT-1 (ref. ${ }^{50}$ ) in mice decreases atherosclerotic burden. Cumulatively, these studies implicate hypoxia as a driver of macrophage phenotype in atherosclerosis progression, highlighting that HIF- $1 \alpha$ activation and increased glycolysis are central to this pathologic response.

Macrophage death and efferocytosis. Prolonged defects in cholesterol efflux and/or free-cholesterol esterification by the ER can ultimately lead to macrophage death in plaques. ER stress has been documented in human and mouse atherosclerotic-plaque macrophages, particularly in the advanced stages of disease, and decreasing ER stress can mitigate atherosclerosis ${ }^{97,98}$. ER stress leads to the induction of the unfolded-protein response, which reestablishes homeostasis in the ER, thereby maintaining functions in folding and secretion of proteins, calcium storage, and lipid synthesis for membrane biogenesis or energy storage. However, if prolonged, ER stress can trigger apoptosis through the ER-resident protein IRE1 or downstream effectors such as $\mathrm{CHOP}^{5}$. In advanced plaques, clearance of apoptotic cells by surrounding macrophages can become compromised as these cells undergo metabolic dysfunction. Efficient clearance of apoptotic cells by macrophages requires intact lysosomal function and lipid metabolism to cope with the dramatic increase in lipid, protein, nucleotide, and carbohydrate content that occurs after the ingestion of apoptotic bodies. Thus, the combination of defective macrophage cholesterol efflux, enhanced apoptotic cell death, and defective efferocytosis by plaque macrophages can result in secondary necrosis and the release of cellular components into the plaque milieu, effects that in turn are thought to contribute to the formation of the lipid-rich, acellular necrotic core that characterizes vulnerable plaques.

Efferocytosis is dependent on a coordinated metabolic cellular response. In vitro, FAO is enhanced during efferocytosis, and altering the mitochondrial membrane potential by glucose deprivation or providing excess glucose can increase and decrease efferocytosis, respectively ${ }^{99}$. Thus, the mitochondrial membrane potential within macrophages is a critical determinant of phagocytic capacity and continued apoptotic cell uptake ${ }^{99}$. Mitochondrial morphology exists in dynamic flux, changing rapidly through fission or fusion in response to external stimuli and cellular metabolic needs. In settings of nutrient deprivation, increased demand for mitochondrial respiration leads to mitochondrial fusion, which is associated with increased ATP production and resistance to autophagy ${ }^{100}$. In atherosclerosis, mitochondrial fission is required in response to apoptotic cell uptake for effective efferocytosis by macrophages in the atherosclerotic plaque ${ }^{101}$. Interestingly, M[IL-4] macrophages, which have high OXPHOS, have a greater efferocytosis capacity than do $\mathrm{M}[\mathrm{LPS}+\mathrm{IFN} \gamma]$ macrophages, thus suggesting that this difference in metabolism may determine macrophage efferocytosis ability in atherosclerotic plaques. In agreement with this possibility, factors associated with M[LPS+IFN $\gamma$ ] macrophages, such as oxidative stress and hypoxia, inhibit efferocytosis in atherosclerotic plaques ${ }^{92,102}$.

Hyperglycemia. Hyperglycemia associated with type 1 or type 2 diabetes mellitus is an independent risk factor for atherosclerosis and cardiovascular events ${ }^{103}$, and is associated with elevated inflammatory monocyte and macrophage activation and hematopoiesis ${ }^{104}$. 
Type 1 diabetes-induced hyperglycemia results in proliferation and expansion of bone marrow myeloid cell progenitors and release of inflammatory monocytes into the circulation, coinciding with impairment of atherosclerosis resolution, which is mitigated with treatment for hyperglycemia ${ }^{104}$. Interestingly, overexpression of the glucose transporter GLUT1 in myeloid cells has been found to increase glycolysis and flux through the PPP, coupled with a compensatory decrease in FAO; however, this overexpression alone does not induce cytokine production ${ }^{105}$. Furthermore, in Apoe $^{-/-}$mice, myeloid-specific overexpression of GLUT-1 does not promote atherosclerosis. Thus, macrophage glycolytic flux alone is not sufficient to drive atherosclerosis, and the atheropromoting macrophage response to a hyperglycemic systemic milieu is driven by factors beyond increased rates of glycolysis. The metabolic changes in myeloid cells in the diabetic setting remain an area of active investigation.

\section{Epigenetic and metabolic memory shape macrophage responses}

Although metabolic modulation is integral to the macrophage activation response, recent discoveries have also unveiled its role in 'learned' innate immune functional responses. Macrophages exposed to acute or chronic stimuli, both in vitro and in vivo, can remodel their chromatin to mount a 'learned' response to subsequent stimulation with the same or different stimuli, in a process termed innate immune memory. These memory responses are characterized phenotypically by cellular inflammatory responses that are partly regulated by changes in metabolic machinery. Although these effects have predominately been defined in the context of pathogen response, emerging evidence suggests that innate immune memory also regulates the metabolic and effector response to sterile inflammatory disease states, such as atherosclerosis.

Two categories of innate immune memory responses to pathogenic stimuli have been described: 'trained' or 'tolerant' effector responses. Innate immune training, which results in a potentiated inflammatory response of monocytes and macrophages, occurs in response to pathogenic stimuli such as bacillus Calmette-Guéri vaccination and $\beta$-glucan (Fig. 3). Innate immune tolerance, which results in a dampened response to secondary stimuli, occurs after exposure to LPS. The divergent responses of training and tolerance are facilitated in part by metabolic cellular adaptations. For example, $\beta$-glucan-induced training is accompanied by a metabolic switch from OXPHOS to aerobic glycolysis via activation of the mTOR-HIF1 $\alpha$ pathway. Epigenetic analysis of cells after $\beta$-glucan stimulation has revealed an increase in trimethylated histone $\mathrm{H} 3 \mathrm{~K} 4$ and acetylated histone $\mathrm{H} 3 \mathrm{~K} 27$ in the promoter region of EIF4EBP1, the main target of mTOR, and highly increased expression of genes involved in the mTOR signaling and glycolytic pathways ${ }^{106}$. The increase in acetylated histone H3K27 in $\beta$-glucan-trained monocytes has been linked to a decrease in the $\mathrm{NAD}^{+}$-dependent class III histone deacetylase sirtuin-1, and early treatment with the sirtuin- 1 activator resveratrol during $\beta$-glucan training partially decreases IL-6 production ${ }^{106}$. Integration of the transcriptome and metabolome of $\beta$-glucan-trained monocytes and macrophages has also revealed upregulation of metabolic pathways, including glucose metabolism, glutaminolysis, and cholesterol synthesis ${ }^{26,106}$. In contrast, macrophages undergoing innate immune tolerance after exposure to LPS exhibit a metabolic switch from glycolysis to OXPHOS and an increased $\mathrm{NAD}^{+} / \mathrm{NADH}$ ratio, thus resulting in activation of the histone deacetylases sirtuin-1 and sirtuin- 6 and consequent inhibition inflammatory-gene transcription $^{107}$. Collectively, these findings have established the paradigm that the innate immune memory of macrophages is linked to their metabolic programs and can influence their subsequent inflammatory response to microbial and probably endogenous inflammatory ligands.
Innate immune memory occurs not only in monocytes and macrophages but also within myeloid progenitor populations in the bone marrow. $\beta$-glucan-treated mice, up to $28 \mathrm{~d}$ after treatment, show increased HSPC populations, including long-term hematopoietic stem cells ${ }^{108}$. RNA-seq of long-term hematopoietic stem cells $7 \mathrm{~d}$ after $\beta$-glucan injection has identified glycolysis, cholesterol biosynthesis, and the mevalonate pathway as functions overrepresented among significantly upregulated genes, whereas the expression of genes involved in cholesterol efflux is decreased. Within the bone marrow, IL-1 $\beta$ is increased, and increased numbers of CD131 ${ }^{+}$ (the GM-CSF receptor) HSPCs have been observed. Treatment with an IL-1-receptor antagonist or blockade of GM-CSF restricted the $\beta$-glucan-induced expansion of HSPCs, as did inhibition of glycolysis with $2 \mathrm{DG}$ or inhibition of the cholesterol-biosynthesis pathway with statin therapy.

In monocytes, activation of the cholesterol-biosynthesis pathway, but not cholesterol synthesis itself, has been found to be essential for the induction of trained immunity ${ }^{109}$. Statins prevent training induced by $\beta$-glucan and bacillus Calmette-Guéri, thus blunting both upregulation of cytokine production after secondary LPS stimulation and increased glycolysis. The addition of exogenous mevalonate, the metabolite directly downstream of HMG-CoA reductase (the target of the statin class of drugs), is sufficient to rescue the $\beta$-glucan innate immune training phenotype in the presence of a statin, and mevalonate alone induces a trained immunity phenotype through epigenetic reprogramming, enhanced glycolysis, and mTOR signaling. The ability of mevalonate to induce innate immune training indicates that this response is not restricted to microbial stimuli and that factors associated with sterile inflammatory insults, such as those that occur in atherosclerosis and other chronic inflammatory conditions, are likely to induce similar effector and metabolic responses that heighten inflammation.

Recent studies have implicated two microenvironmental factors of atherosclerosis, namely oxLDL and hypercholesterolemia, as sterile drivers of innate immune training. Human monocytes exposed to oxLDL ${ }^{53}$ and then re-stimulated with TLR2 (Pam3Cys) or TLR4 (LPS) agonists exhibit increased expression of inflammatory cytokines (IL-6, TNF $\alpha$, IL-8, and MCP-1), and this effect is reversed by treatment with a methylation inhibitor, thus implicating epigenetic reprogramming in this response. In an independent study, human monocytes trained with oxLDL for $5 \mathrm{~d}$ and then stimulated with LPS have also been found to exhibit enhanced IL-6 and TNF- $\alpha$ responses ${ }^{110}$, which are abrogated by treatment with a recombinant IL-1R antagonist. Notably, statin treatment of oxLDLtrained monocytes also blunts the immune-training phenotype by mitigating the enhanced glycolytic and proinflammatory-cytokine response to LPS re-stimulation ${ }^{109}$. In vivo, a hypercholesterolemic environment has been found to drive innate immune training in mice ${ }^{110}$. Monocytes isolated from $L d l r^{-1-}$ mice fed a western diet (WD) for 4 weeks showed enhanced cytokine and chemokine production when stimulated ex vivo with various TLR agonists. Furthermore, in vivo treatment of mice fed a WD for 4 weeks with LPS resulted in higher levels of serum inflammatory cytokines than were observed in chow-diet-fed mice. In agreement with findings from previous studies, the WD increased myelopoiesis, including levels of the myeloid progenitor GMP population, which showed priming of inflammatory genes and a downregulation of cholesterol-biosynthesis pathways in RNA-seq analysis. Notably, switching the WD-fed mice back to chow diet for 4 weeks normalized the systemic inflammatory milieu but did not normalize the enhanced monocyte response to ex vivo TLR stimulation. RNA-sequencing and assay for transposase-accessible chromatin using sequencing (ATAC-sequencing, which identifies changes in open and accessible chromatin) revealed that the WD effectively reprogrammed GMPs at the transcriptional and epigenetic levels. Notably, treatment of mice with an IL-1R antagonist, or deficiency of the NLRP3 
a

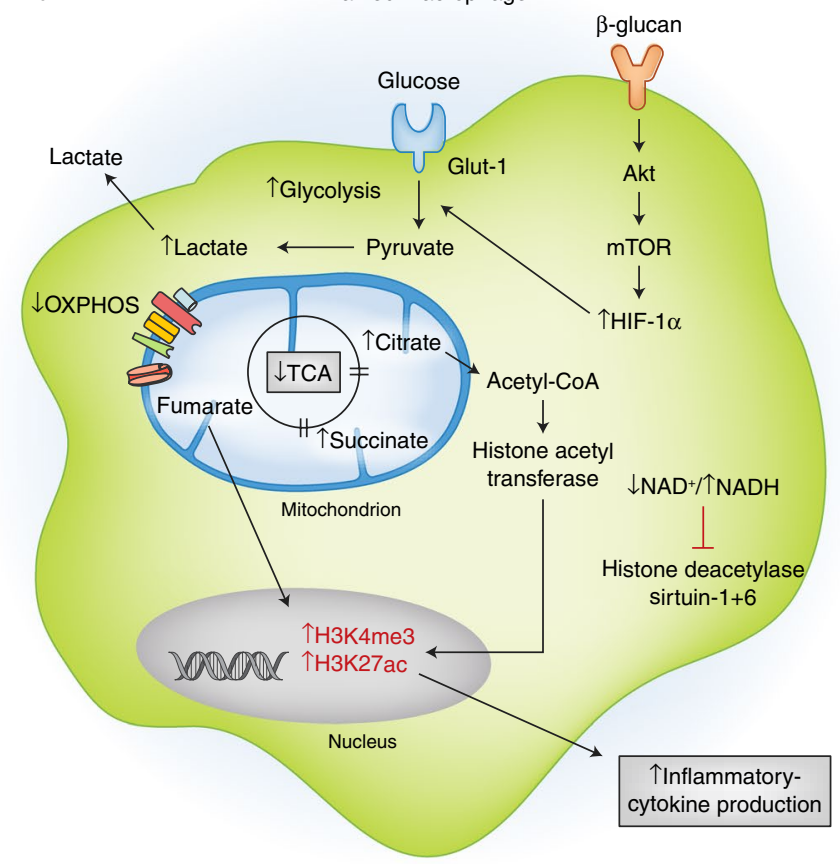

b

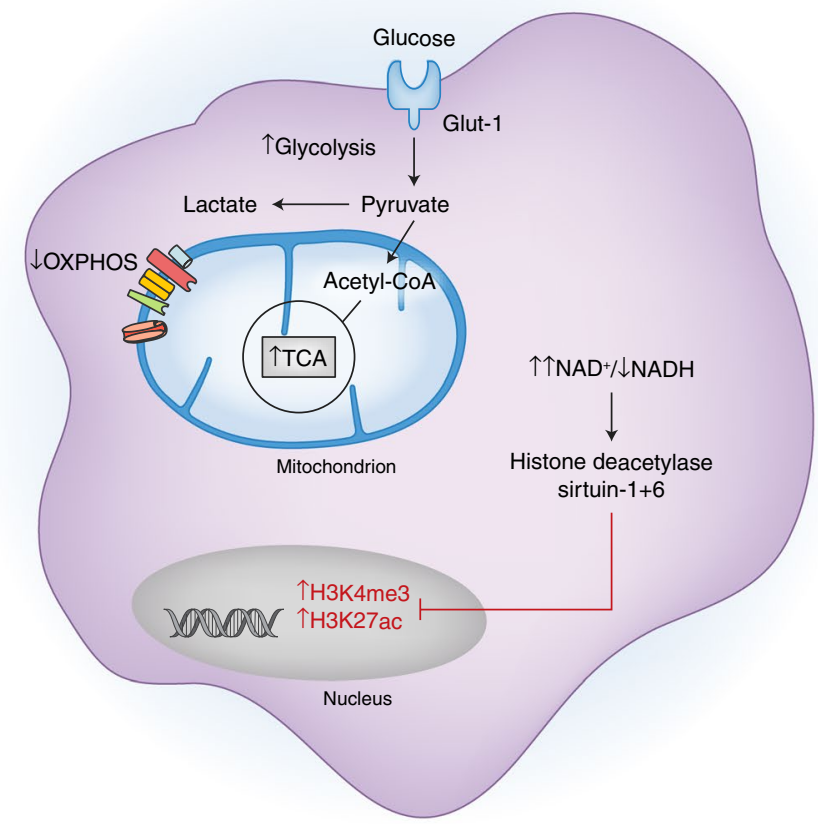

Fig. 3 | Epigenetic alterations that shape the macrophage immune response. Stimuli that activate immune cells and induce metabolic rewiring are also capable of inducing epigenetic remodeling through histone modifications, thereby resulting in innate immune memory. a, In trained macrophages (e.g., those stimulated with bacillus Calmette-Guéri or $\beta$-glucan), a broken TCA cycle results in the accumulation of citrate and succinate. These metabolites activate histone-modifying enzymes, such as histone acetyltransferase, thereby increasing K4-trimethylated histone $\mathrm{H} 3$ (H3K4me3) and K27-acetylated histone $\mathrm{H} 3(\mathrm{H} 3 \mathrm{~K} 27 \mathrm{ac})$ at promoter sites of genes encoding inflammatory cytokines and subsequently resulting in an increased inflammatory response. Additionally, a decreased ratio of $\mathrm{NAD}^{+}$to $\mathrm{NADH}$ in trained cells inhibits the activity of $\mathrm{NAD}^{+}$-dependent histone deacetylases sirtuin- 1 and sertuin- 6 , thus supporting the increased inflammatory response. $\mathbf{b}$, This response is absent in nontrained macrophages, which have lower basal levels of H3K4me3 and H3K27ac, owing to an intact TCA cycle, which prevents the accumulation of citrate and succinate, and an elevated NAD ${ }^{+} / \mathrm{NADH}$ ratio, which results in activation of sirtuin-1 and sirtuin-6. Akt; protein kinase B. Credit: Kim Caesar/Springer Nature

inflammasome, which regulates IL-1 $\beta$ production, abrogated the enhanced cytokine response of WD-trained monocytes.

In clinical studies, circulating monocytes from patients with atherosclerosis have also been found to have an enhanced inflammatory signature that is associated with epigenetic remodeling, which may also be connected to metabolic alterations. For example, an enhanced inflammatory-cytokine response to TLR agonists also occurs in patients with either high lipoprotein(a) levels ${ }^{111}$ or established atherosclerosis ${ }^{112}$. Monocytes isolated from patients with symptomatic atherosclerosis elicit a stronger cytokine response to ex vivo challenge with LPS and P3C than do monocytes from controls. A subsequent analysis of mRNA from whole-blood circulating cells has revealed higher expression of glycolytic (HK2, PFKFB3, PKM, and PDHA1), TCA-cycle (ASL and MDH1), and PPP (TALDO1) genes, as compared with expression in controls ${ }^{112}$. Collectively, these studies in mice and humans have unveiled the potent effects of innate immune training on circulating monocytes and hematopoietic progenitors that may contribute to atherogenesis. Furthermore, the role of IL- $1 \beta$ in macrophage innate immune training in response to a hypercholesterolemic environment ${ }^{110}$ is noteworthy, given the recent findings from the CANTOS trial that therapeutic targeting of IL- $1 \beta$ with the monoclonal antibody canakinumab decreases the rate of recurrent cardiovascular events in patients with previous myocardial infarction ${ }^{14}$, as well as lung cancer incidence and mortality ${ }^{113}$. These findings raise the possibility that part of the beneficial effects of blocking IL-1 $\beta$ signaling in cardiovascular disease may derive from the ability of this treatment to reverse myeloid cell epigenetic reprogramming during inflammation, as well as subsequent heightened immune responses.

\section{Summary and future directions}

Much work remains to be done to unravel the intertwined and dynamic metabolic and epigenetic landscapes that determine immunologic function in myeloid cells as these cells are exposed to microbial stimuli and unique tissue microenvironments in health and disease. In atherosclerosis, it is clear that macrophages exhibit metabolic rewiring in response to dyslipidemia and environmental factors (hypoxia, dying cells, and cytokines) that lead to metabolic heterogeneity among plaque macrophages. These metabolic changes are likely to be dynamic, because the microenvironment evolves during both plaque progression and treatment of disease. Although not discussed in this review, metabolic reprogramming is also ongoing in the other cell types in plaques, including $\mathrm{T}$ cells, dendritic cells, endothelial cells, and smooth muscle cells, and these changes in turn affect macrophage metabolic programs through nutrient competition, extracellular accumulation of metabolic byproducts, and cell-cell signaling and interactions.

In the field of basic macrophage biology, there is a need for better delineation of metabolic adaptations and the roles of specific metabolites in regulating distinct macrophage effector responses, such as phagocytosis, cytokine production, cholesterol efflux, autophagy, and extracellular matrix synthesis and degradation. Although these processes are often studied in isolation in cultured cells, there is also a need to understand how macrophages coordinate their metabolic needs when they must exert multiple effector responses simultaneously. Furthermore, beyond single environmental stimulatory agents, there is a need to understand how macrophages behave in vivo, where the microenvironment is more complex, and where macrophages experience multiple stimuli 
concurrently. Further study, particularly at the single-cell level, will be needed to determine how macrophages prioritize the different signals that they are exposed to and adopt metabolic signatures that fulfill these different needs.

From an atherosclerosis perspective, it will be important to better understand how the metabolic signatures of monocytes and macrophages change during early atherogenesis, and to determine the point at which these programs become maladaptive and disease promoting. Such an understanding will require studying macrophage metabolism across multiple stages of atherosclerosis, including early foam cell lesions, advanced plaques, and, importantly, plaques vulnerable to rupture, which present the highest risk for myocardial infarction. To date, metabolic studies of macrophages taken from plaques have been limited because their isolation is challenging, yet these cells offer metabolic clues for understanding the switch from functional to dysfunctional metabolic programs.

There is intense interest in exploiting the plasticity of macrophage metabolism to rebalance inflammatory responses and restore protective immune functions in atherosclerosis. A better understanding of the specific metabolic rewiring of activated macrophages during both progression and regression of atherosclerosis will be of fundamental importance in identifying novel therapeutic approaches. Evidence from mouse models of atherosclerosis regression has already indicated that lowering apoB-lipoprotein production or raising circulating HDL levels shifts the macrophage plaque phenotype toward that of M[IL-4]-like tissue-reparative macrophages ${ }^{66-68}$ and that this shift requires ongoing recruitment of Ly6 $\mathrm{c}^{\mathrm{hi}}$ monocytes into plaques ${ }^{114}$. The identification of drugs targeting metabolic intermediates or pathways that could reorient macrophage effector functions to restore homeostasis to the artery wall are needed. In addition, as more evidence emerges that epigenetic memory and metabolic memory are interconnected, drugs that alter epigenetic marks on chromatin are being explored as potential tools to manipulate macrophage inflammatory responses. The increasing availability of small molecules capable of manipulating metabolic routes and epigenetic marks is opening new avenues for the discovery and potential therapeutic application of small molecules in atherosclerosis and beyond.

Received: 30 March 2018; Accepted: 18 April 2018; Published online: 18 May 2018

\section{References}

1. Andrejeva, G. \& Rathmell, J. C. Similarities and distinctions of cancer and immune metabolism in inflammation and tumors. Cell Metab. 26, 49-70 (2017).

2. Lachmandas, E. et al. Microbial stimulation of different Toll-like receptor signalling pathways induces diverse metabolic programmes in human monocytes. Nat. Microbiol. 2, 16246 (2016).

3. Liu, L. et al. Proinflammatory signal suppresses proliferation and shifts macrophage metabolism from Myc-dependent to HIF1 $\alpha$-dependent. Proc. Natl Acad. Sci. USA 113, 1564-1569 (2016).

4. Stienstra, R., Netea-Maier, R. T., Riksen, N. P., Joosten, L. A. B. \& Netea, M. G. Specific and complex reprogramming of cellular metabolism in myeloid cells during innate immune responses. Cell Metab. 26, 142-156 (2017).

5. Moore, K. J., Sheedy, F. J. \& Fisher, E. A. Macrophages in atherosclerosis: a dynamic balance. Nat. Rev. Immunol. 13, 709-721 (2013)

6. Park, Y. M., Febbraio, M. \& Silverstein, R. L. CD36 modulates migration of mouse and human macrophages in response to oxidized LDL and may contribute to macrophage trapping in the arterial intima. J. Clin. Invest. 119, 136-145 (2009).

7. van Gils, J. M. et al. The neuroimmune guidance cue netrin-1 promotes atherosclerosis by inhibiting the emigration of macrophages from plaques. Nat. Immunol. 13, 136-143 (2012).

8. Tabas, I. \& Lichtman, A. H. Monocyte-macrophages and T cells in atherosclerosis. Immunity 47, 621-634 (2017).

9. Tabas, I. \& Glass, C. K. Anti-inflammatory therapy in chronic disease: challenges and opportunities. Science 339, 166-172 (2013).

10. Robbins, C. S. et al. Local proliferation dominates lesional macrophage accumulation in atherosclerosis. Nat. Med. 19, 1166-1172 (2013).
11. Tabas, I. Macrophage death and defective inflammation resolution in atherosclerosis. Nat. Rev. Immunol. 10, 36-46 (2010).

12. Grundy, S. M. et al. Implications of recent clinical trials for the National Cholesterol Education Program Adult Treatment Panel III guidelines. Arterioscler. Thromb. Vasc. Biol. 24, e149-e161 (2004).

13. Libby, P., Ridker, P. M. \& Hansson, G. K. Progress and challenges in translating the biology of atherosclerosis. Nature 473, 317-325 (2011).

14. Ridker, P. M. et al. Antiinflammatory therapy with canakinumab for atherosclerotic disease. N. Engl. J. Med. 377, 1119-1131 (2017).

15. O'Neill, L. A., Kishton, R. J. \& Rathmell, J. A guide to immunometabolism for immunologists. Nat. Rev. Immunol. 16, 553-565 (2016).

16. Christofk, H. R. et al. The M2 splice isoform of pyruvate kinase is important for cancer metabolism and tumour growth. Nature 452, 230-233 (2008).

17. Haschemi, A. et al. The sedoheptulose kinase CARKL directs macrophage polarization through control of glucose metabolism. Cell Metab. 15, 813-826 (2012).

18. Ecker, J. et al. Induction of fatty acid synthesis is a key requirement for phagocytic differentiation of human monocytes. Proc. Natl Acad. Sci. USA 107, 7817-7822 (2010).

19. Ménégaut, L., Thomas, C., Lagrost, L. \& Masson, D. Fatty acid metabolism in macrophages: a target in cardio-metabolic diseases. Curr. Opin. Lipidol. 28, 19-26 (2017).

20. Van den Bossche, J., O’Neill, L. A. \& Menon, D. Macrophage immunometabolism: where are we (going)? Trends Immunol. 38, 395-406 (2017).

21. Palsson-McDermott, E. M. et al. Pyruvate kinase M2 regulates Hif- $1 \alpha$ activity and IL- $1 \beta$ induction and is a critical determinant of the warburg effect in LPS-activated macrophages. Cell Metab. 21, 65-80 (2015).

22. Tannahill, G. M. et al. Succinate is an inflammatory signal that induces IL-1 $\beta$ through HIF-1 $\alpha$. Nature 496, 238-242 (2013).

23. Jha, A. K. et al. Network integration of parallel metabolic and transcriptional data reveals metabolic modules that regulate macrophage polarization. Immunity 42, 419-430 (2015).

24. Infantino, V. et al. The mitochondrial citrate carrier: a new player in inflammation. Biochem. J. 438, 433-436 (2011).

25. Tallam, A. et al. Gene regulatory network inference of immunoresponsive gene 1 (IRG1) identifies interferon regulatory factor 1 (IRF1) as its transcriptional regulator in mammalian macrophages. PLoS One 11, e0149050 (2016)

26. Arts, R. J. et al. Glutaminolysis and fumarate accumulation integrate immunometabolic and epigenetic programs in trained immunity. Cell Metab. 24, 807-819 (2016).

27. Posokhova, E. N., Khoshchenko, O. M., Chasovskikh, M. I., Pivovarova, E. N. \& Dushkin, M. I. Lipid synthesis in macrophages during inflammation in vivo: effect of agonists of peroxisome proliferator activated receptors alpha and gamma and of retinoid X receptors. Biochemistry (Mosc.) 73, 296-304 (2008).

28. Feingold, K. R. et al. Mechanisms of triglyceride accumulation in activated macrophages. J. Leukoc. Biol. 92, 829-839 (2012).

29. Everts, B. et al. TLR-driven early glycolytic reprogramming via the kinases TBK1-IKKe supports the anabolic demands of dendritic cell activation. Nat. Immunol. 15, 323-332 (2014).

30. Ham, M. et al. Macrophage glucose-6-phosphate dehydrogenase stimulates proinflammatory responses with oxidative stress. Mol. Cell. Biol. 33, 2425-2435 (2013).

31. Wallace, C. \& Keast, D. Glutamine and macrophage function. Metabolism 41, 1016-1020 (1992).

32. Huang, S. C. et al. Cell-intrinsic lysosomal lipolysis is essential for alternative activation of macrophages. Nat. Immunol. 15, 846-855 (2014).

33. Tan, Z. et al. Pyruvate dehydrogenase kinase 1 participates in macrophage polarization via regulating glucose metabolism. J. Immunol. 194, 6082-6089 (2015).

34. Nomura, M. et al. Fatty acid oxidation in macrophage polarization. Nat. Immunol. 17, 216-217 (2016).

35. Van den Bossche, J. et al. Mitochondrial dysfunction prevents repolarization of inflammatory macrophages. Cell Rep. 17, 684-696 (2016).

36. Pesce, J. T. et al. Arginase-1-expressing macrophages suppress Th2 cytokinedriven inflammation and fibrosis. PLoS Pathog. 5, e1000371 (2009).

37. Boutens, L. et al. Unique metabolic activation of adipose tissue macrophages in obesity promotes inflammatory responses. Diabetologia 61, 942-953 (2018).

38. Huang, S. C. et al. Metabolic reprogramming mediated by the mTORC2IRF4 signaling axis is essential for macrophage alternative activation. Immunity 45, 817-830 (2016).

39. Tavakoli, S., Zamora, D., Ullevig, S. \& Asmis, R. Bioenergetic profiles diverge during macrophage polarization: implications for the interpretation of 18F-FDG PET imaging of atherosclerosis. J. Nucl. Med. 54, 1661-1667 (2013). 
40. Izquierdo, E. et al. Reshaping of human macrophage polarization through modulation of glucose catabolic pathways. J. Immunol. 195, 2442-2451 (2015).

41. Na, Y. R. et al. Proteomic analysis reveals distinct metabolic differences between granulocyte-macrophage colony stimulating factor (GM-CSF) and macrophage colony stimulating factor (M-CSF) grown macrophages derived from murine bone marrow cells. Mol. Cell. Proteomics 14, 2722-2732 (2015)

42. Cheng, S. C. et al. Broad defects in the energy metabolism of leukocytes underlie immunoparalysis in sepsis. Nat. Immunol. 17, 406-413 (2016).

43. Folco, E. J. et al. Hypoxia but not inflammation augments glucose uptake in human macrophages: Implications for imaging atherosclerosis with ${ }^{18}$ fluorine-labeled 2-deoxy-D-glucose positron emission tomography. J. Am. Coll. Cardiol. 58, 603-614 (2011).

44. Ip, W. K. E., Hoshi, N., Shouval, D. S., Snapper, S. \& Medzhitov, R. Anti-inflammatory effect of IL-10 mediated by metabolic reprogramming of macrophages. Science 356, 513-519 (2017).

45. Murphy, A. J., Dragoljevic, D. \& Tall, A. R. Cholesterol efflux pathways regulate myelopoiesis: a potential link to altered macrophage function in atherosclerosis. Front. Immunol. 5, 490 (2014).

46. Dutta, P. et al. Myocardial infarction accelerates atherosclerosis. Nature 487, 325-329 (2012).

47. Heidt, T. et al. Chronic variable stress activates hematopoietic stem cells. Nat. Med. 20, 754-758 (2014).

48. Murphy, A. J. \& Tall, A. R. Disordered haematopoiesis and atherothrombosis. Eur. Heart J. 37, 1113-1121 (2016).

49. Gautier, E. L. et al. HDL and Glut1 inhibition reverse a hypermetabolic state in mouse models of myeloproliferative disorders. J. Exp. Med. 210, 339-353 (2013).

50. Sarrazy, V. et al. Disruption of Glut1 in hematopoietic stem cells prevents myelopoiesis and enhanced glucose flux in atheromatous plaques of Apo $E^{-/-}$mice. Circ. Res. 118, 1062-1077 (2016).

51. Shirai, T. et al. The glycolytic enzyme PKM2 bridges metabolic and inflammatory dysfunction in coronary artery disease. J. Exp. Med. 213, 337-354 (2016).

52. Lee, S. J. et al. Oxidized low-density lipoprotein stimulates macrophage ${ }^{18}$ F-FDG uptake via hypoxia-inducible factor- $1 \alpha$ activation through Nox2-dependent reactive oxygen species generation. J. Nucl. Med. 55, 1699-1705 (2014)

53. Bekkering, S. et al. Oxidized low-density lipoprotein induces long-term proinflammatory cytokine production and foam cell formation via epigenetic reprogramming of monocytes. Arterioscler. Thromb. Vasc. Biol. 34, 1731-1738 (2014).

54. Wang, Y., Wang, G. Z., Rabinovitch, P. S. \& Tabas, I. Macrophage mitochondrial oxidative stress promotes atherosclerosis and nuclear factor- $\kappa$ B-mediated inflammation in macrophages. Circ. Res. 114, 421-433 (2014).

55. Stewart, C. R. et al. CD36 ligands promote sterile inflammation through assembly of a Toll-like receptor 4 and 6 heterodimer. Nat. Immunol. 11, 155-161 (2010).

56. Miller, Y. I. et al. Toll-like receptor 4-dependent and -independent cytokine secretion induced by minimally oxidized low-density lipoprotein in macrophages. Arterioscler. Thromb. Vasc. Biol. 25, 1213-1219 (2005).

57. Sheedy, F. J. et al. CD36 coordinates activation of the NLRP3 inflammasome by facilitating the intracellular nucleation of soluble to particulate ligands in sterile inflammation. Nat. Immunol. 14, 812-820 (2013).

58. Duewell, P. et al. NLRP3 inflammasomes are required for atherogenesis and activated by cholesterol crystals. Nature 464, 1357-1361 (2010).

59. Haneklaus, M. \& O'Neill, L. A. NLRP3 at the interface of metabolism and inflammation. Immunol. Rev. 265, 53-62 (2015).

60. Zhu, X. et al. Macrophage ABCA1 reduces MyD88-dependent Toll-like receptor trafficking to lipid rafts by reduction of lipid raft cholesterol. J. Lipid Res. 51, 3196-3206 (2010).

61. Mogilenko, D. A. et al. Endogenous apolipoprotein A-I stabilizes ATP-binding cassette transporter A1 and modulates Toll-like receptor 4 signaling in human macrophages. FASEB J. 26, 2019-2030 (2012).

62. Yvan-Charvet, L. et al. Increased inflammatory gene expression in ABC transporter-deficient macrophages: free cholesterol accumulation, increased signaling via toll-like receptors, and neutrophil infiltration of atherosclerotic lesions. Circulation 118, 1837-1847 (2008).

63. Tawakol, A. et al. HIF- $1 \alpha$ and PFKFB3 mediate a tight relationship between proinflammatory activation and anerobic metabolism in atherosclerotic macrophages. Arterioscler. Thromb. Vasc. Biol. 35, 1463-1471 (2015).

64. Khallou-Laschet, J. et al. Macrophage plasticity in experimental atherosclerosis. PLoS One 5, e8852 (2010).

65. Chinetti-Gbaguidi, G. et al. Human atherosclerotic plaque alternative macrophages display low cholesterol handling but high phagocytosis because of distinct activities of the PPAR $\gamma$ and LXR $\alpha$ pathways. Circ. Res. 108, 985-995 (2011).
66. Feig, J. E. et al. Reversal of hyperlipidemia with a genetic switch favorably affects the content and inflammatory state of macrophages in atherosclerotic plaques. Circulation 123, 989-998 (2011).

67. Feig, J. E. et al. HDL promotes rapid atherosclerosis regression in mice and alters inflammatory properties of plaque monocyte-derived cells. Proc. Natl Acad. Sci. USA 108, 7166-7171 (2011).

68. Rayner, K. J. et al. Antagonism of miR-33 in mice promotes reverse cholesterol transport and regression of atherosclerosis. J. Clin. Invest. 121, 2921-2931 (2011).

69. Cardilo-Reis, L. et al. Interleukin-13 protects from atherosclerosis and modulates plaque composition by skewing the macrophage phenotype. EMBO Mol. Med. 4, 1072-1086 (2012).

70. Kadl, A. et al. Identification of a novel macrophage phenotype that develops in response to atherogenic phospholipids via Nrf2. Circ. Res. 107, 737-746 (2010).

71. Yvan-Charvet, L., Wang, N. \& Tall, A. R. Role of HDL, ABCA1, and ABCG1 transporters in cholesterol efflux and immune responses. Arterioscler. Thromb. Vasc. Biol. 30, 139-143 (2010).

72. Karunakaran, D. et al. Macrophage mitochondrial energy status regulates cholesterol efflux and is enhanced by anti-miR33 in atherosclerosis. Circ. Res. 117, 266-278 (2015).

73. McCarthy, C. et al. Macrophage PPAR gamma co-activator-1 alpha participates in repressing foam cell formation and atherosclerosis in response to conjugated linoleic acid. EMBO Mol. Med. 5, 1443-1457 (2013)

74. Vilne, B. et al. Network analysis reveals a causal role of mitochondrial gene activity in atherosclerotic lesion formation. Atherosclerosis 267 39-48 (2017).

75. Ouimet, M. et al. miRNA targeting of oxysterol-binding protein-like 6 regulates cholesterol trafficking and efflux. Arterioscler. Thromb. Vasc. Biol. 36, 942-951 (2016).

76. Rayner, K. J. et al. MiR-33 contributes to the regulation of cholesterol homeostasis. Science 328, 1570-1573 (2010).

77. Ouimet, M. et al. microRNA-33 regulates macrophage autophagy in atherosclerosis. Arterioscler. Thromb. Vasc. Biol. 37, 1058-1067 (2017).

78. Ouimet, M. et al. Mycobacterium tuberculosis induces the miR-33 locus to reprogram autophagy and host lipid metabolism. Nat. Immunol. 17, 677-686 (2016)

79. Dávalos, A. et al. miR-33a/b contribute to the regulation of fatty acid metabolism and insulin signaling. Proc. Natl Acad. Sci. USA 108, 9232-9237 (2011).

80. Rotllan, N., Ramírez, C. M., Aryal, B., Esau, C. C. \& Fernández-Hernando, C. Therapeutic silencing of microRNA-33 inhibits the progression of atherosclerosis in Ldlr ${ }^{-/-}$mice: brief report. Arterioscler. Thromb. Vasc. Biol. 33, 1973-1977 (2013).

81. Ouimet, M. et al. MicroRNA-33-dependent regulation of macrophage metabolism directs immune cell polarization in atherosclerosis. J. Clin. Invest. 125, 4334-4348 (2015).

82. Vendrov, A. E. et al. Atherosclerosis is attenuated by limiting superoxide generation in both macrophages and vessel wall cells. Arterioscler. Thromb. Vasc. Biol. 27, 2714-2721 (2007).

83. Yu, E. P. K. et al. Mitochondrial respiration is reduced in atherosclerosis, promoting necrotic core formation and reducing relative fibrous cap thickness. Arterioscler. Thromb. Vasc. Biol. 37, 2322-2332 (2017).

84. Shimada, K. et al. Oxidized mitochondrial DNA activates the NLRP3 inflammasome during apoptosis. Immunity 36, 401-414 (2012).

85. Guo, H., Callaway, J. B. \& Ting, J. P. Inflammasomes: mechanism of action, role in disease, and therapeutics. Nat. Med. 21, 677-687 (2015).

86. Rongvaux, A. et al. Development and function of human innate immune cells in a humanized mouse model. Nat. Biotechnol. 32, 364-372 (2014).

87. White, M. J. et al. Apoptotic caspases suppress mtDNA-induced STINGmediated type I IFN production. Cell 159, 1549-1562 (2014).

88. West, A. P. et al. Mitochondrial DNA stress primes the antiviral innate immune response. Nature 520, 553-557 (2015).

89. Sergin, I., Evans, T. D., Bhattacharya, S. \& Razani, B. Hypoxia in plaque macrophages: a new danger signal for interleukin- $1 \beta$ activation? Circ. Res. 115, 817-820 (2014)

90. Aarup, A. et al. Hypoxia-inducible factor- $1 \alpha$ expression in macrophages promotes development of atherosclerosis. Arterioscler. Thromb. Vasc. Biol. 36, 1782-1790 (2016)

91. Sluimer, J. C. et al. Hypoxia, hypoxia-inducible transcription factor, and macrophages in human atherosclerotic plaques are correlated with intraplaque angiogenesis. J. Am. Coll. Cardiol. 51, 1258-1265 (2008).

92. Marsch, E. et al. Reversal of hypoxia in murine atherosclerosis prevents necrotic core expansion by enhancing efferocytosis. Arterioscler. Thromb. Vasc. Biol. 34, 2545-2553 (2014).

93. Corcoran, S. E. \& O'Neill, L. A. HIF1 $\alpha$ and metabolic reprogramming in inflammation. J. Clin. Invest. 126, 3699-3707 (2016). 
94. Folco, E. J., Sukhova, G. K., Quillard, T. \& Libby, P. Moderate hypoxia potentiates interleukin-1 $\beta$ production in activated human macrophages. Circ. Res. 115, 875-883 (2014).

95. Parathath, S. et al. Hypoxia is present in murine atherosclerotic plaques and has multiple adverse effects on macrophage lipid metabolism. Circ. Res. 109, 1141-1152 (2011).

96. Chandel, N. S. et al. Reactive oxygen species generated at mitochondrial complex III stabilize hypoxia-inducible factor-1alpha during hypoxia: a mechanism of $\mathrm{O}_{2}$ sensing. J. Biol. Chem. 275, 25130-25138 (2000).

97. Tufanli, O. et al. Targeting IRE1 with small molecules counteracts progression of atherosclerosis. Proc. Natl Acad. Sci. USA 114, E1395-E1404 (2017)

98. Erbay, E. et al. Reducing endoplasmic reticulum stress through a macrophage lipid chaperone alleviates atherosclerosis. Nat. Med. 15, 1383-1391 (2009).

99. Park, D. et al. Continued clearance of apoptotic cells critically depends on the phagocyte Ucp2 protein. Nature 477, 220-224 (2011).

100. Liesa, M. \& Shirihai, O. S. Mitochondrial dynamics in the regulation of nutrient utilization and energy expenditure. Cell Metab. 17, 491-506 (2013).

101. Wang, Y. et al. Mitochondrial fission promotes the continued clearance of apoptotic cells by macrophages. Cell 171, 331-345.e322 (2017).

102. Schrijvers, D. M., De Meyer, G. R., Kockx, M. M., Herman, A. G. \& Martinet, W. Phagocytosis of apoptotic cells by macrophages is impaired in atherosclerosis. Arterioscler. Thromb. Vasc. Biol. 25, 1256-1261 (2005).

103. Bornfeldt, K. E. \& Tabas, I. Insulin resistance, hyperglycemia, and atherosclerosis. Cell Metab. 14, 575-585 (2011)

104. Nagareddy, P. R. et al. Hyperglycemia promotes myelopoiesis and impairs the resolution of atherosclerosis. Cell Metab. 17, 695-708 (2013).

105. Nishizawa, T. et al. Testing the role of myeloid cell glucose flux in inflammation and atherosclerosis. Cell Rep. 7, 356-365 (2014).

106. Cheng, S. C. et al. mTOR- and HIF- $1 \alpha$-mediated aerobic glycolysis as metabolic basis for trained immunity. Science 345, 1250684 (2014).

107. Liu, T. F., Vachharajani, V. T., Yoza, B. K. \& McCall, C. E. NAD ${ }^{+}$-dependent sirtuin 1 and 6 proteins coordinate a switch from glucose to fatty acid oxidation during the acute inflammatory response. J. Biol. Chem. 287, 25758-25769 (2012)
108. Mitroulis, I. et al. Modulation of myelopoiesis progenitors is an integral component of trained immunity. Cell 172, 147-161.e112 (2018).

109. Bekkering, S. et al. Metabolic induction of trained immunity through the mevalonate pathway. Cell 172, 135-146.e139 (2018).

110. Christ, A. et al. Western diet triggers NLRP3-dependent innate immune reprogramming. Cell 172, 162-175.e114 (2018).

111. van der Valk, F. M. et al. Oxidized phospholipids on lipoprotein(a) elicit arterial wall inflammation and an inflammatory monocyte response in humans. Circulation 134, 611-624 (2016).

112. Bekkering, S. et al. Innate immune cell activation and epigenetic remodeling in symptomatic and asymptomatic atherosclerosis in humans in vivo. Atherosclerosis 254, 228-236 (2016).

113. Ridker, P. M. et al. Effect of interleukin-1 $\beta$ inhibition with canakinumab on incident lung cancer in patients with atherosclerosis: exploratory results from a randomised, double-blind, placebo-controlled trial. Lancet 390, 1833-1842 (2017).

114. Rahman, K. et al. Inflammatory Ly6 $\mathrm{C}^{\text {hi }}$ monocytes and their conversion to M2 macrophages drive atherosclerosis regression. J. Clin. Invest. 127, 2904-2915 (2017).

\section{Acknowledgements}

Work in the laboratory of K.J.M. related to this review was supported by the National Institutes of Health (R35HL135799 and P01HL131481). G.J.K. was supported by a doctoral foreign study award from the Canadian Institutes of Health Research. E.E. was supported by an European Research Council Starting Grant (336643).

\section{Competing interests}

The authors declare no competing interests.

\section{Additional information}

Reprints and permissions information is available at www.nature.com/reprints.

Correspondence should be addressed to K.J.M.

Publisher's note: Springer Nature remains neutral with regard to jurisdictional claims in published maps and institutional affiliations. 